\title{
Curcumin prevents renal cell apoptosis in acute kidney injury in a rat model of dry-heat environment heatstroke via inhibition of the mitochondrial apoptotic pathway
}

\author{
YIN-HUI ZHAO ${ }^{1,2^{*}}$, CAI-FU SHEN ${ }^{1 *}$, YAN KANG $^{3 *}, \mathrm{AO} \mathrm{QI}^{1}$, \\ WEN-JUAN XU ${ }^{1}$, WEN-HUI SHI ${ }^{1}$ and JIANG-WEI LIU ${ }^{1}$
}

\author{
${ }^{1}$ Key Laboratory of The Special Environmental Medicine of Xinjiang, General Hospital of Xinjiang Military Region \\ of The PLA, Urumqi, Xinjiang 830000; ${ }^{2}$ Emergency Critical Department, Shanghai General Hospital, Shanghai 200080; \\ ${ }^{3}$ Department of Imaging Medicine The 69240 Army Hospital of PLA, Urumqi, Xinjiang 830000, P.R. China
}

Received April 2, 2020; Accepted October 20, 2020

DOI: $10.3892 /$ etm.2020.9558

\begin{abstract}
Heatstroke is a life-threatening illness that is characterised by a core body temperature $>40^{\circ} \mathrm{C}$ and central nervous system dysfunction. Acute kidney injury (AKI) is a common complication of heatstroke, and the mitochondrial apoptotic pathway has been demonstrated to be one of the leading causes of tissue damage and cell death in AKI. Curcumin is a phenol that is extracted from turmeric and demonstrates anti-apoptotic properties. To test if curcumin can protect the kidney from injury caused by heat stress, the effect of curcumin administration on renal injury and apoptosis of renal tissue was examined in a rat model of dry-heat environment. A total of 50 Sprague-Dawley rats were randomly divided into five groups $(\mathrm{n}=10)$ : Standard temperature control, dry-heat control and curcumin treatment groups $(50,100$ and $200 \mathrm{mg} / \mathrm{kg}$ groups). After exposure to a dry-heat environment for $150 \mathrm{~min}$, the rats were anesthetized and euthanized. Blood, urine and renal tissue were collected to quantify the expression of specific mitochondrial apoptosis-related molecules. Curcumin pre-treatment decreased blood urea nitrogen and serum creatinine, urinary kidney injury molecule-1, and neutrophil gelatinase-associated lipocalin levels compared with the dry-heat control group. Curcumin was also revealed to downregulate c-Jun $\mathrm{N}$-terminal kinases (JNK),
\end{abstract}

Correspondence to: Professor Jiang-Wei Liu, Key Laboratory of The Special Environmental Medicine of Xinjiang, General Hospital of Xinjiang Military Region of The PLA, 359 Youhao North Road, Urumqi, Xinjiang 830000, P.R. China

E-mail: ljw273273@163.com

*Contributed equally

Abbreviations: AKI, acute kidney injury

Key words: heatstroke, acute kidney injury, curcumin, mitochondrial apoptotic pathway, rat model cytochrome $c$, caspase-3 and caspase- 9 expression upon treatment with 100 and $200 \mathrm{mg} / \mathrm{kg}$ curcumin, which may result in inhibition of the mitochondrial apoptotic pathway in renal cells. The current study revealed that Curcumin may to have potential for preventing heatstroke-induced AKI.

\section{Introduction}

Heatstroke is characterised by a core body temperature $>40^{\circ} \mathrm{C}$ and central nervous system dysfunction resulting in delirium, convulsions or coma (1). Heatstroke is a life-threatening condition that is often accompanied by organ injury and a poor prognosis including sequela or even mortality (2). Acute kidney injury (AKI) is a common complication of heatstroke. Increased pathogenesis of heatstroke-associated AKI is likely due to decreased perfusion caused by dehydration and subsequent hypovolemia, direct thermal injury, rhabdomyolysis-associated myoglobinuria and systemic inflammatory response syndrome (3). In addition, the release of inflammatory factors and direct heat damage can induce apoptosis in cells, as observed in a baboon animal model (4) of heatstroke (5).

Currently, the pathologic process responsible for tissue and cell damage induced by heatstroke is not clearly understood. In addition, no effective clinical methods have been developed for early diagnosis, and affordable treatment options are also lacking, resulting in elevated rates of mortality in patients with heatstroke.

Heat is one of the most influential external factors that affects cellular function and structure (6). Rapidly increasing ambient temperatures can lead to extensive cell degeneration and necrosis in tissues, and when core body temperatures exceed $41.6-42^{\circ} \mathrm{C}$ for $>45 \mathrm{~min}$, cells undergo apoptosis (7). These extreme temperatures can induce destruction of cell structures and necrosis within minutes (8). Sakaguchi et al (9) demonstrated that exposure of a rat model of heat shock to temperatures of $41.5^{\circ} \mathrm{C}$ for $2 \mathrm{~h}$ induced apoptosis in healthy tissue cells. Therefore, cellular apoptosis may be one of the mechanisms responsible for the development of renal injury induced by heatstroke. Therefore, it is important to identify compounds that are capable of reversing or inhibiting these apoptotic pathways, thereby limiting AKI in patients with heatstroke. 
Curcumin is a phenol that is extracted from turmeric. Studies have demonstrated that curcumin exhibits a wide range of biological functions, including inhibition of cell proliferation, antioxidant properties, anti-apoptotic effects and scavenging of oxygen free radicals (10-12). In addition, multiple studies have demonstrated a protective effect of curcumin on AKI induced by a number of different factors including toxic drugs (13-15).

It can therefore be hypothesised that curcumin exhibits a protective effect in renal injury caused by heat stress. In the current study, the previously described dry-heat environment protocol was used to establish a rat model of heatstroke, determine the effect of curcumin pre-treatment on renal pathological changes and explore the possible underlying mechanism governing this interaction.

\section{Materials and methods}

Animals. A total of 50 male Sprague-Dawley (SD) rats (65-70 days old; 190-220 g) were purchased from the Experimental Animal Center of Xinjiang Medical University. Animals were housed in cages in groups (five rats per cage) at $20 \pm 2^{\circ} \mathrm{C}$ and $40-50 \%$ humidity with a $12 \mathrm{~h}$ light/dark cycle. The rats were fed a standard pellet diet and provided with water ad libitum. The present study was approved by the ethical committee of the General Hospital of Xinjiang Military Region of the PLA. Animal care and experiments were conducted according to the National Science Council guidelines.

Establishment of a rat model of heatstroke, curcumin pre-treatment and collection of blood, urine and kidney tissue samples. The $50 \mathrm{SD}$ rats were randomly divided into five groups $(\mathrm{n}=10)$ : Normal temperature control (NT control), dry-heat control (DH control), and curcumin treatment groups including 50, 100 and $200 \mathrm{mg} / \mathrm{kg}$ groups, the curcumin concentrations were based on the previous relevant research $(16,17)$. The control rats were pretreated with $0.9 \%$ saline by gavage while experimental rats were administered curcumin orally. All rats were pretreated once a day for seven consecutive days. Curcumin was dissolved in $0.5 \%$ sodium carboxymethyl cellulose $(\mathrm{CMCNa})$ solution prior to administration.

The dry-heat heatstroke rat model was replicated after 7 days of pre-treatment from our previous study (18). The NT control group rats were incubated at room temperature $\left(20 \pm 2^{\circ} \mathrm{C}\right)$ with a humidity of $40-50 \%$. The remaining rats were incubated in the dry-heat environment (The Simulated Climate Cabin for Special Environment of Northwest of China, Urumqi, China) at a temperature of $41^{\circ} \mathrm{C} \pm 0.5^{\circ} \mathrm{C}$ and $10 \pm 1 \%$ humidity. The rat core body temperature was monitored (using a thermometer to measure rectal body temperature) every $30 \mathrm{~min}$. The rats were removed from the experimental cabin following $150 \mathrm{~min}$ of incubation and were anesthetized by intraperitoneal injection

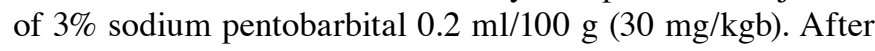
the animals were anesthetized, blood was collected from the inferior vena cava for analysis of blood indicators, and urine was collected by puncturing the bladder to assess the renal injury index. Renal tissue was stored at $-80^{\circ} \mathrm{C}$ for subsequent analysis. After the specimen was extracted, the rats were euthanized using cervical dislocation.
Biochemical analysis. Serum was separated via centrifugation at $1,006.2 \mathrm{xg}$ for $10 \mathrm{~min}$ at $4^{\circ} \mathrm{C}$ and stored at $-20^{\circ} \mathrm{C}$ for analyses of creatinine and blood urea nitrogen (BUN) levels using a fully automatic biochemical analyser (Mindray BS-180; Shenzhen Mindray Bio-Medical Electronics Co., Ltd.).

Measurement of kidney injury molecule-1 (KIM-1) and neutrophil gelatinase-associated lipocalin (NGAL) levels in urine. The expression of KIM-1 (cat. no. MKM100) and NGAL (cat. no. MLCN20) in urine were quantified using commercial ELISA kits (R\&D Systems, Inc.) according to the manufacturer's instructions.

Western blot analysis. The kidney samples were ground with liquid nitrogen followed by lysis with a Cell Lysis Buffer (cat. no. ab152163; Abcam) for $2 \mathrm{~h}$ on ice. The lysates were centrifuged at $4,024.8 \times \mathrm{g}$ for $20 \mathrm{~min}$ at $4^{\circ} \mathrm{C}$, and supernatants were collected and stored at $-80^{\circ} \mathrm{C}$ in Eppendorf tubes. Samples were mixed with $2 \mathrm{X}$ loading buffer (Abcam) and boiled for $8 \mathrm{~min}$ before they were subjected to electrophoresis. Protein samples (120 ng) were quantified using a BCA protein assay kit (Pierce ${ }^{\mathrm{TM}}$ BCA Protein Assay kit; Thermo Fisher Scientific, Inc.). Electrophoresis was then performed at $60 \mathrm{~V}$ (5\% stacking gel) and then at $100 \mathrm{~V}(10 \%$ separating gel) for $1.5 \mathrm{~h}$, and electrotransferred for 20-30 min according to different molecular weights (sample amount, $0.5 \mu \mathrm{g}$ ). The PVDF membrane was blocked using $5 \%$ non-fat milk powder for $2 \mathrm{~h}$ at room temperature, followed by incubation with the following primary antibodies: Cytochrome $c$, cat. no. 4272; JNK, cat. no. 9252; caspase-9, cat. no. 9504 and caspase-3, cat. no. 9662; all from Cell Signaling Technology, Inc.) at $4^{\circ} \mathrm{C}$ overnight. Secondary antibodies (goat anti-rabbit IgG H\&L, cat. no. ab6721; rabbit anti-mouse IgG H\&L, cat. no. ab6728; both from Abcam) were then added, and the membrane was incubated at room temperature for $1 \mathrm{~h}$. The target band was detected by chemiluminescence (ChemDoc-IT ${ }^{\circledR} 510$ Imager, Ultra-Violet Products Ltd.), and the protein was semi-quantified using Visionworks LS (version 8.1.2; Ultra-Violet Products Ltd.) following analysis of the grey intensity.

TUNEL staining for detection of apoptotic cells. Apoptotic cells (fixed in $10 \%$ methanol at $4^{\circ} \mathrm{Cfor} 24 \mathrm{~h}$ ) in the kidney sections were detected using a TUNEL assay kit (In Situ Cell Death Detection kit; Roche Applied Science) according to the manufacturer's instructions. For each study group, the apoptosis index was calculated using a 10x field of view. Apoptotic cells were observed via an optical microscope (magnification, $\mathrm{x} 400$ ) and imaged, Each group was captured in 10 fields. Apoptotic cells were manually identified by their specific morphological characteristics (presence of apoptotic bodies, chromatin condensation, marginalisation and membrane lysis). The apoptosis index was calculated as follows: Apoptosis cells/total cells within a high-power field.

Changes in morphology observed under an electron microscope. Kidney specimens were cut into 2-mm sized fragments and fixed in $2.5 \%$ glutaraldehyde at room temperature in $0.1 \mathrm{M}$ phosphate buffer overnight. The tissues were washed thrice in $0.1 \mathrm{M}$ phosphate buffer and fixed with $1 \%$ osmium tetroxide in phosphate buffer for $1 \mathrm{~h}$ at $4^{\circ} \mathrm{C}$. The fixed tissues were then 
A

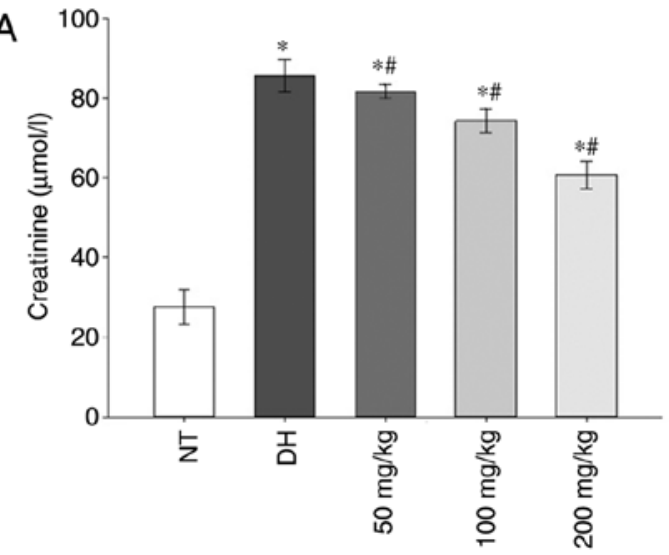

B

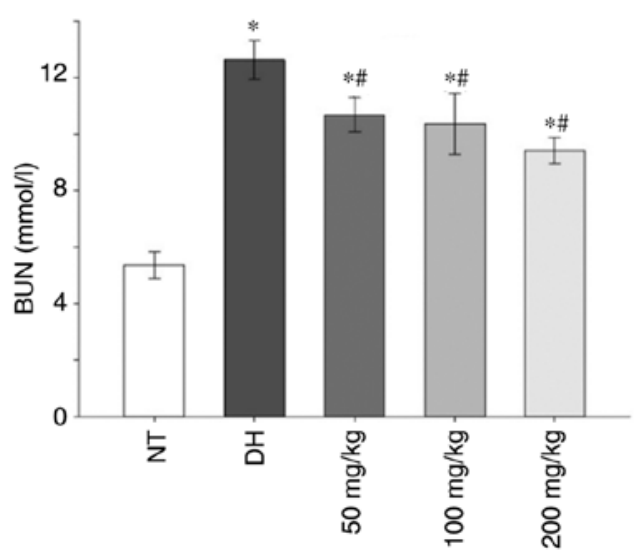

Figure 1. Blood serum analysis. Concentration of (A) creatinine and (B) BUN in blood serum samples in the control, dry heat or curcumin treatment groups. Compared with the NT control group, the concentration of creatinine and BUN in serum samples was significantly increased in the DH and all curcumin treatment groups. Pre-treatment with increasing concentrations of curcumin resulted in a significant decrease in BUN and creatinine levels compared with the $\mathrm{DH}$ control. Values are expressed as means $\pm \mathrm{SE}(\mathrm{n}=10)$ and analysed using one-way ANOVA. ${ }^{*} \mathrm{P}<0.05$ vs. NT. ${ }^{\sharp} \mathrm{P}<0.05$ vs. DH. BUN, blood urea nitrogen.
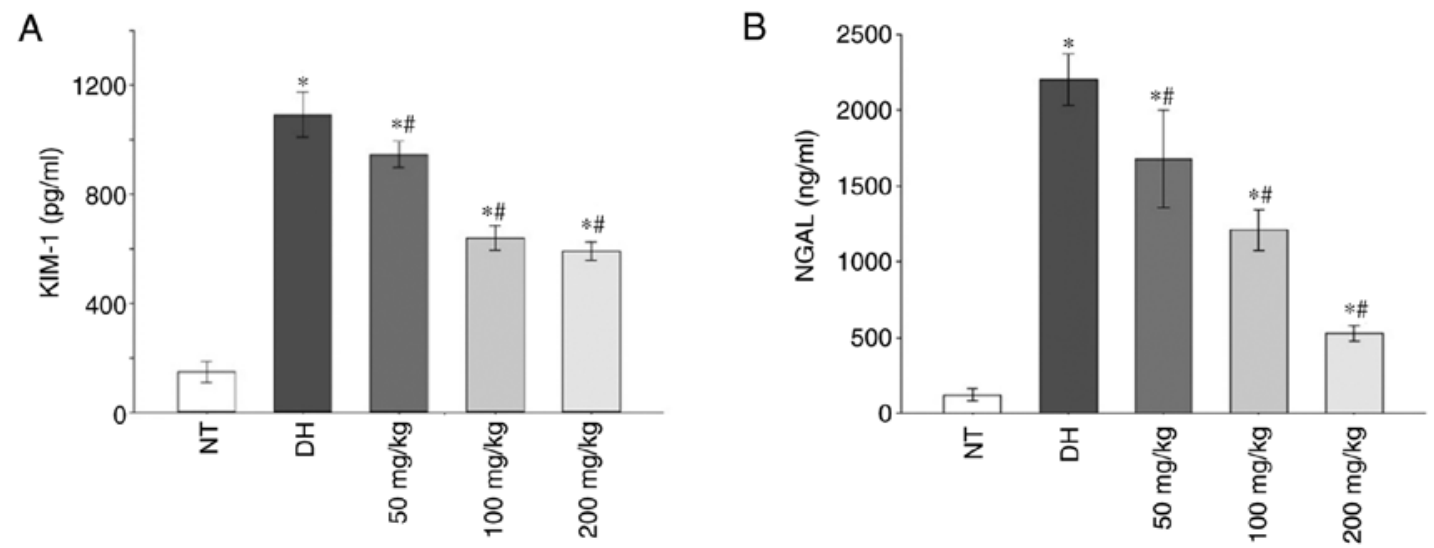

Figure 2. Expression of Acute kidney injury markers. Levels of (A) KIM-1 and (B) NGAL in urine samples in the control, dry heat or curcumin treatment groups. KIM-1 and NGAL increased significantly in the DH and all curcumin treatment groups compared with the NT control group. At all different curcumin concentrations, the expression of KIM-1 and NGAL were significantly decreased compared with the DH control group. Values are expressed as means \pm SE $(\mathrm{n}=10)$ and analysed using one-way ANOVA. ${ }^{*} \mathrm{P}<0.05$ vs. NT. " $\mathrm{P}<0.05$ vs. DH. KIM-1, kidney injury molecule-1; NGAL, neutrophil gelatinase-associated lipocalin.

washed thrice in $0.1 \mathrm{M}$ phosphate buffer. The specimens were dehydrated using a graded series of ethanol $(50,70,80,90,95$ and $100 \%$ ) for 15-20 min at each step and transferred to absolute acetone for $20 \mathrm{~min}$. The specimens were then placed in a 1:1 mixture of absolute acetone and a Spurr resin mixture for $1 \mathrm{~h}$ at room temperature and then transferred to a 1:3 mixture of the same solution overnight. The next day, specimens were placed in capsules containing embedding medium and heated at $70^{\circ} \mathrm{C}$ for $\sim 9 \mathrm{~h}$. The sections were then sequentially stained with uranyl acetate and alkaline lead citrate at $25^{\circ} \mathrm{C}$ for $15 \mathrm{~min}$ each and observed under a transmission electron microscope (JEM-1230; JEOL, Ltd.).

Statistical analysis. In the current study, repeated measurement data are presented as the mean \pm SD $(n=10$ in each group). One-way ANOVA was used for comparison between groups, and Bonfferoni was used as a post-hoc test. All statistical analysis was performed by SPSS software (version 21.0; IBM Corp.). $\mathrm{P}<0.05$ was considered to indicate a statistically significant difference.

\section{Results}

Compared with the NT control group, the concentration of creatinine and BUN in serum samples was significantly increased in the DH and all curcumin treatment groups $(\mathrm{P}<0.05)$. However, pre-treatment with increasing concentrations of curcumin resulted in a significant decrease in BUN and creatinine levels compared with the $\mathrm{DH}$ control $(\mathrm{P}<0.05$; Fig. 1A and B).

Additionally, in the DH and all curcumin treatment groups, the levels of early renal injury markers, namely KIM-1 and NGAL, increased significantly compared with the NT control group. At all different curcumin concentrations, the expression of KIM-1 and NGAL were significantly decreased compared with the $\mathrm{DH}$ control group $(\mathrm{P}<0.05$; Fig. $2 \mathrm{~A}$ and $\mathrm{B})$.

Following incubation for $150 \mathrm{~min}$ in a dry-heat environment, in the DH and all curcumin treatment groups, the expression of cytochrome $c$ (Cyt $c$ ), JNK, caspase-3 and caspase- 9 was revealed to be increased compared with the NT control group. However, 100 and $200 \mathrm{mg} / \mathrm{kg}$ curcumin 
A

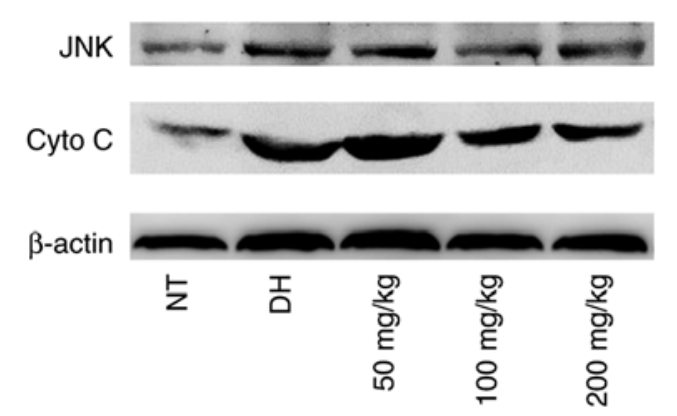

C

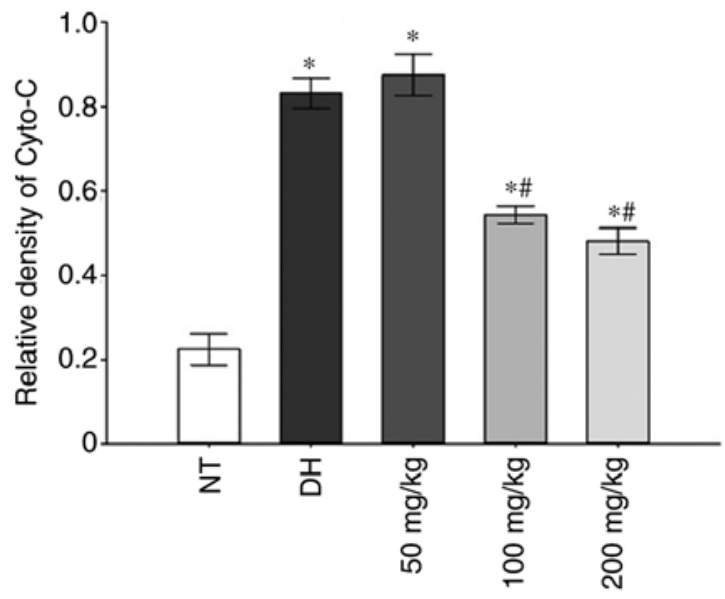

E

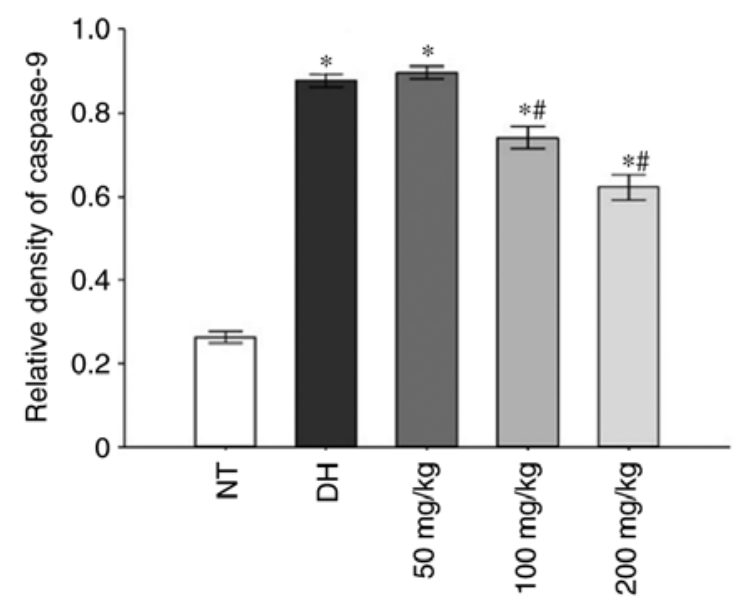

B

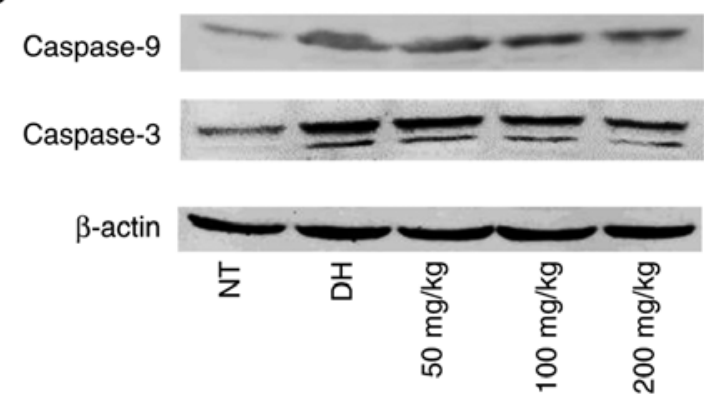

D

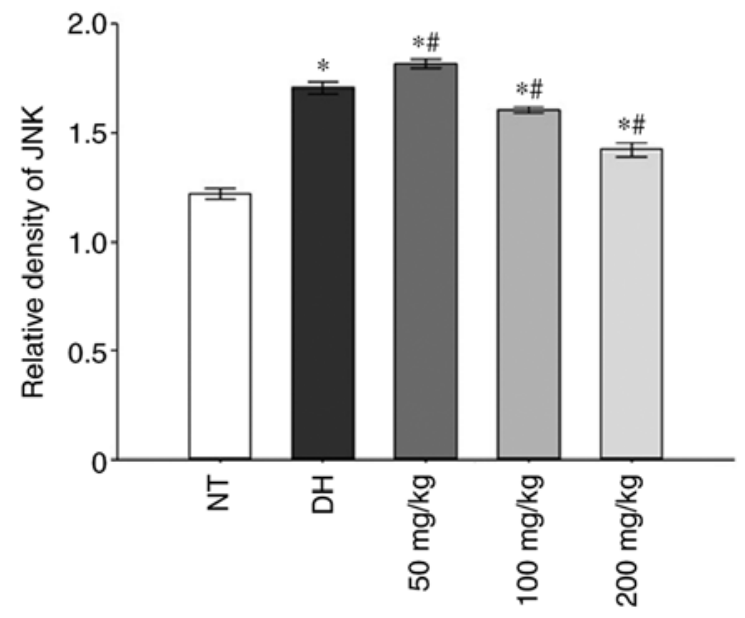

$\mathrm{F}$

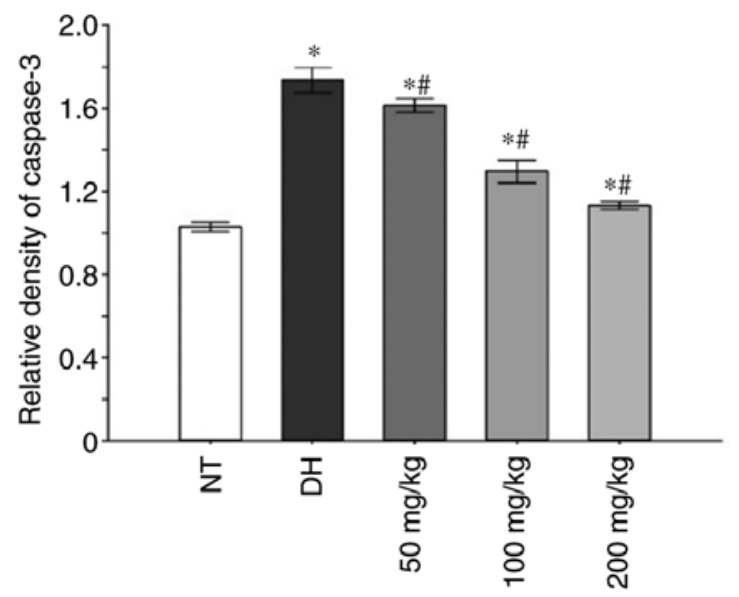

Figure 3. Expression of apoptotic-related markers. Changes in the expression of (A) Cyto-C and JNK and (B) caspase-9 and caspase-3 in the control, dry heat or curcumin treatment groups. The expression of (C) Cyto-C, (D) JNK, (E) caspase-3 and (F) caspase-9 was revealed to be increased in the DH and all curcumin treatment groups compared with the NT control group. However, 100 and $200 \mathrm{mg} / \mathrm{kg}$ curcumin pe-treatment group caused the expression of these apoptosis-related proteins to significantly decrease. Values are expressed as means \pm SE $(n=10)$ and analysed using one-way ANOVA. *P<0.05 vs. NT. "P<0.05 vs. DH. Cyto C, cytochrome $c$.

pre-treatment group caused the expression of these apoptosis-related proteins to significantly decrease (Fig. 3).

Paraffin sections of renal tissues were stained using TUNEL. The renal tissues obtained from the NT control group rats did not appear to exhibit significant apoptosis (apoptosis index, $0.55+0.071 \%$ ), whereas those from the $\mathrm{DH}$ and all curcumin treatment groups indicated significantly higher levels of apoptosis in the renal tubular cells compared with the NT group (apoptosis index, $5.5+0.48 \%$; $\mathrm{P}<0.05$ ). The level of apoptosis observed in the renal tissue of rats treated with
$50 \mathrm{mg} / \mathrm{kg}$ curcumin (apoptosis index, 5.1+0.37\%) was significantly lower compared with the $\mathrm{DH}$ control group $(\mathrm{P}<0.05)$. Similarly, the apoptosis index determined for the renal tissue in rats treated with either $100 \mathrm{mg} / \mathrm{kg}(2.05+0.37 \%)$ or $200 \mathrm{mg} / \mathrm{kg}$ $(1.33+0.20 \%)$ of curcumin was also significantly decreased compared with the DH control group ( $\mathrm{P}<0.05$; Fig. 4).

Electron microscopy revealed severe mitochondrial damage within the $\mathrm{DH}$ control group, which was characterised by mitochondrial swelling and vacuolisation, as well as disappearance of the mitochondrial cristae (Fig. 5). Alternatively, 


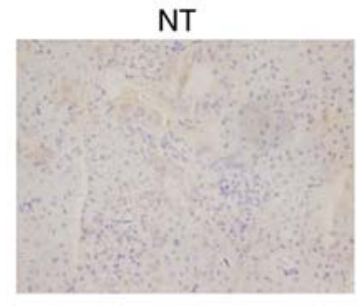

$50 \mathrm{mg} / \mathrm{kg}$

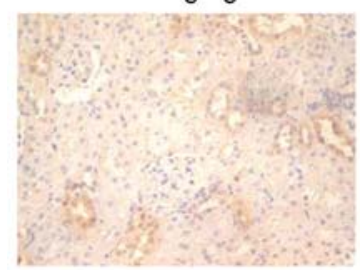

$200 \mathrm{mg} / \mathrm{kg}$
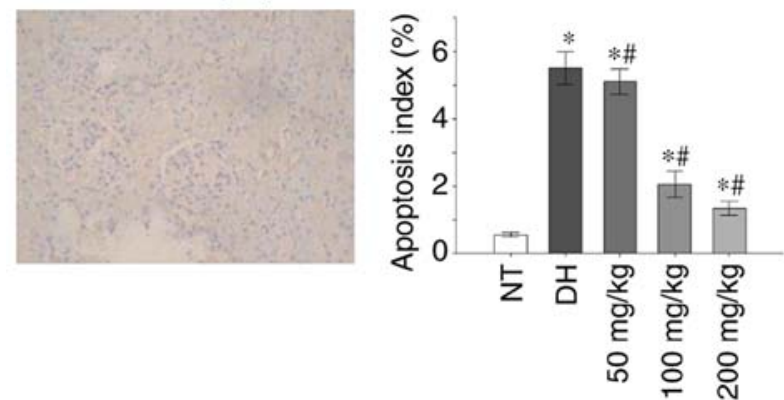

Figure 4. TUNEL staining and apoptosis index of renal tissue sections (magnification, $\mathrm{x} 400$ ). ${ }^{*} \mathrm{P}<0.05$ vs. NT. ${ }^{\#} \mathrm{P}<0.05$ vs. $\mathrm{DH}$.

compared with the DH control group, samples from rats treated with $50 \mathrm{mg} / \mathrm{kg}$ of curcumin demonstrated markedly reduced mitochondrial damage and mitochondrial swelling. In the animals treated with 100 and $200 \mathrm{mg} / \mathrm{kg}$ of curcumin, the mitochondrial swelling was even further reduced, and mitochondria vacuolisation was nearly invisible (Fig. 5).

\section{Discussion}

Increasing evidence has suggested that apoptosis may serve an essential role in the pathological process of heatstroke $(19,20)$. In recent years, researchers have indicated that heatstroke regulates apoptosis by regulating the expression of specific caspases (21). Hsu et al (22) demonstrated that in a heat stress model of corneal cells, cell death was directly related to the heat-induced expression of caspase- 8 and caspase-9, as well as the activation of specific mitochondrial pathways. In addition, Milleron and Bratton (23) demonstrated that inhibition of caspase activity could significantly reduce heat-induced apoptosis. Further research has demonstrated that the direct influence of heat on cells, including cellular damage, production of oxygen metabolism products and production of proteinases and various cytokines that are released during the heat shock process, can activate or inhibit specific signal transduction pathways $(24,25)$, thereby mediating the survival or death of cells.

Apoptosis is characterised by a series of morphological changes, including membrane blebbing, cell shrinkage, chromatin condensation and DNA fragmentation, followed by rapid

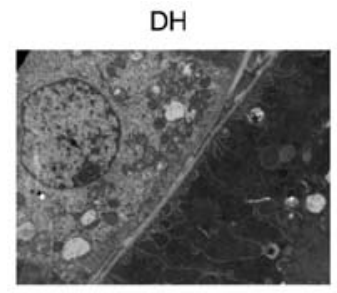

$100 \mathrm{mg} / \mathrm{kg}$

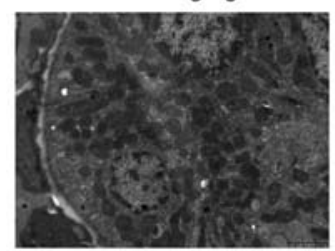

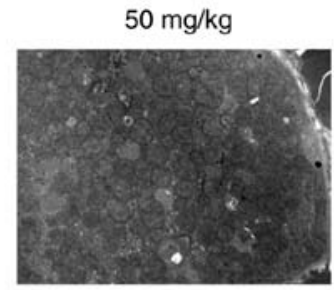

$200 \mathrm{mg} / \mathrm{kg}$

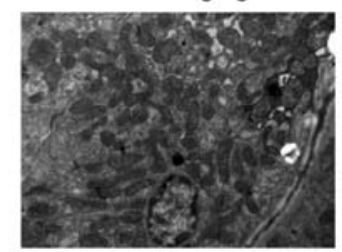

Figure 5. Electron microscopy (magnification, $\mathrm{x} 4,000$ ). Changes in cellular morphology observed under an electron microscope in the dry heat or curcumin treatment groups.

engulfment of the dead cell by neighbouring cells, without rupture of the cell membrane (26). Mitochondria regulate apoptosis through the activation of a variety of death stimuli; therefore, mitochondrial dysfunction serves a prominent function in apoptosis (27). Mitochondrial dysfunction leads to release of Cyt $c$ from the mitochondrial membrane space into the cytoplasm where it serves a vital role in mediating apoptosis $(28,29)$. Released Cyt $c$ binds Apoptotic Peptidase Activating Factor 1 and forms an activation complex with caspase-9, which then serves to activate caspase-3 to induce cell apoptosis and resulting in the activation of downstream cascade reactions.

However, apoptosis is regulated by many upstream signalling pathways. MAPKs are considered to be some of the most important signalling molecules in the transmission of apoptotic signals to the mitochondria (30). Specific MAPK family members, namely JNK and p38, have been determined to be upstream stimulators of classical apoptotic pathways (31). These molecules have been indicated to reduce the expression and activity of the anti-apoptotic Bcl-2 family members by interfering with cellular localisation and dimer formation (32). Alternatively, MAPKs have also been revealed to promote the expression and activity of pro-apoptotic proteins and induce apoptosis via the mitochondrial pathway $(33,34)$.

In the present study, the identification of apoptosis based on the pathological changes in the renal tissue is facile. Following incubation in a dry-heat environment for $150 \mathrm{~min}$, expression of apoptosis-related proteins increased significantly. Therefore, apoptosis may serve an essential role in kidney injury in a dry-heat environment.

To study the benefits of curcumin pre-treatment, rats were pre-treated with different concentrations of curcumin prior to incubation in a dry-heat environment for $150 \mathrm{~min}$ and subsequent changes were observed in the renal tissue, as well as changes in the expression of JNK, Cyt $c$, caspase-3 and caspase-9. The results of electron microscopy revealed that the changes in the renal tissues of the DH control group were noticeable, with mitochondrial injury including swelling and vacuolisation being observed. Increased expression of apoptosis-related proteins, specifically, JNK, Cyt $c$, caspase-3 
and caspase-9 were also observed following incubation in the dry-heat environment. Therefore, mitochondria may serve a significant role in regulating apoptosis within kidney tissues exposed to a dry-heat environment, and inhibiting pathways associated with mitochondrial apoptosis may prove to be beneficial in preventing renal injury following extended exposure to a dry-heat environment. Curcumin pre-treatment (100 and $200 \mathrm{mg} / \mathrm{kg}$ ) resulted in decreased expression of JNK, Cyt $c$, caspase-3, and caspase-9. Electron microscopy revealed that the rats treated with $50 \mathrm{mg} / \mathrm{kg}$ of curcumin demonstrated reduced mitochondrial damage and mitochondrial swelling compared with the DH control. This effect was even more pronounced in tissues from animals treated with $100 \mathrm{mg} / \mathrm{kg}$ or $200 \mathrm{mg} / \mathrm{kg}$ of curcumin, resulting in near elimination of mitochondrial vacuolisation. These findings indicate a dose-dependent protective effect of curcumin on mitochondria.

In conclusion, the results of the current study demonstrated that pre-treatment with curcumin prevents heatstroke-induced AKI in rats. Curcumin (100 and $200 \mathrm{mg} / \mathrm{kg}$ ) groups markedly reduced the expression of specific markers of AKI and apoptosis-related proteins. By reducing the degree of cellular damage through inhibition of the mitochondrial apoptotic pathway, curcumin prevents renal tissue injury induced by heatstroke. Therefore, curcumin may be a potential prophylactic treatment that may prevent the adverse, severe effects of AKI in heatstroke by reducing the degree of cellular damage through inhibition of the mitochondrial apoptotic pathway.

Although some beneficial results have been obtained from the current study, the study has some limitations. A horizontal comparative study was performed. The experiment would have been improved if a vertical comparison at different time points was performed, or relevant factors and mechanism were diversified. Therefore, the correlation between dose and time should be examined in future experiments in order to identify an optimal curcumin concentration and treatment period.

\section{Acknowledgements}

The authors thank Professor Zhao Rong and Professor Xu Qin (Key Laboratory of the Special Environmental Medicine of Xinjiang, General Hospital of Xinjiang Military Region of the PLA) for providing extensive assistance in the experiments presented in this manuscript.

\section{Funding}

The current study was funded by The Clinical Medicine Major Projects of New and High Technology Research of the PLA (grant no. 2010gxjs016).

\section{Availability of data and materials}

The datasets used and/or analyzed during the current study are available from the corresponding author on reasonable request.

\section{Authors' contributions}

YHZ and CFS analyzed and interpreted the data and wrote the manuscript. YK assisted in study design. AQ, WJX and WHS analyzed the data and revised the manuscript. JWL designed the study and drafted the manuscript. All authors read and approved the final manuscript.

\section{Ethics approval and consent to participate}

The present study was approved by the ethical committee of the General Hospital of Xinjiang Military Region of the PLA. Animal care and experiments were conducted according to the National Science Council guidelines.

\section{Patient consent for publication}

Not applicable.

\section{Competing interests}

The authors declare that they have no competing interests.

\section{References}

1. Bouchama A and Knochel JP: Heat stroke. N Engl J Med 346: 1978-1988, 2002.

2. Al Mahri S and Bouchama A: Heatstroke. Handb Clin Neurol 157: 531-545, 2018.

3. Heled Y, Fleischmann C and Epstein Y: Cytokines and their role in hyperthermia and heatstroke. J Basic Clin Physiol Pharmacol 24: 85-96, 2013.

4. Bouchama A, Roberts G, Al Mohanna F, El-Sayed R, Lach B, Chollet-Martin S, Ollivier V, Al Baradei R, Loualich A, Nakeeb S, et al: Inflammatory, hemostatic, and clinical changes in a baboon experimental model for heatstroke. J Appl Physiol (1985) 98: 697-705, 2005

5. Roberts GT, Ghebeh H, Chishti MA, Al-Mohanna F, El-Sayed R, Al-Mohanna F and Bouchama A: Microvascular injury, thrombosis, inflammation, and apoptosis in the pathogenesis of heatstroke: A study in baboon model. Arterioscler Thromb Vasc Biol 28: 1130-1136, 2008.

6. Khogali M: Heat-related illnesses. Middle East J Anaesthesiol 12: 531-572, 1994.

7. Gong L, Zhang Q, Pan X, Chen S, Yang L, Liu B, Yang W, Yu L, Xiao ZX, Feng XH, et al: p53 protects cells from death at the heatstroke threshold temperature. Cell Rep 29: 3693-3707.e5, 2019.

8. Lim CL and Mackinnon LT: The roles of exercise-induced immune system disturbances in the pathology of heat stroke: The dual pathway model of heat stroke. Sports Med 36: 39-64, 2006.

9. Sakaguchi Y, Stephens CL, Makino M, Kaneko T, Strebel FR, Danhauser LL, Jenkins GN and Bull JM: Apoptosis in tumors and normal tissues induced by whole body hyperthermia in rats. Cancer Res 55: 5459-5464, 1995.

10. Unlu A, Nayir E, Dogukan Kalenderoglu M, Kirca O and Ozdogan M: Curcumin Turmeric) and cancer. J BUON 21: 1050-1060, 2016.

11. Anand P, Kunnumakkara AB, Newman RA and Aggarwal BB: Bioavailability of curcumin: Problems and promises. Mol Pharm 4: 807-818, 2007.

12. Mirzaei H, Shakeri A, Rashidi B, Jalili A, Banikazemi Z and Sahebkar A: Phytosomal curcumin: A review of pharmacokinetic, experimental and clinical studies. Biomed Pharmacother 85: 102-112, 2017.

13. He L, Peng X, Zhu J, Liu G, Chen X, Tang C, Liu H, Liu F and Peng Y: Protective effects of curcumin on acute gentamicin-induced nephrotoxicity in rats. Can J Physiol Pharmacol 93: 275-282, 2015.

14. Abdel-Moneim AM, El-Toweissy MY, Ali AM, Awad Allah AA, Darwish HS and Sadek IS: Curcumin ameliorates lead $(\mathrm{Pb}(2+))$-induced hemato-biochemical alterations and renal oxidative damage in a rat model. Biol Trace Elem Res 168: 206-220, 2015.

15. Hismiogullari AA, Hismiogullari SE, Karaca O, Sunay FB, Paksoy S, Can M, Kus I, Seyrek K and Yavuz O: The protective effect of curcumin administration on carbon tetrachloride (CCl4)-induced nephrotoxicity in rats. Pharmacol Rep 67: 410-416, 2015. 
16. Jiang J, Liu J, Li J, Tao L, Wang Z, Yang L, Shi W and Ma N: Effect of curcumin on expressions of CD11b and CD19 in peripheral blood of heat stroke rats in a simulation dry-heat environment. Zhonghua Wei Zhong Bing Ji Jiu Yi Xue 31: 221-224, 2019 (In Chinese)

17. Cao W, Cao JJ, Liu JW, Li JJ, Shen CF, Song LY, Ma N, Shi WH and Xu Q: Effects of curcumin pretreatment on lung injury and HMGB-1 and ICAM-1 mRNA in heat stroke rats in desert dry heat environment. Prog Mod Biomed 18: 652-656, 2018

18. ou Zhou R, Liu JW, Zhang D and Zhang Q: Heatstroke model for desert dry-heat environment and observed organ damage. Am J Emerg Med 32: 573-579, 2014.

19. Zhu YH and Pei ZM: GSK2193874 treatment at heatstroke onset reduced cell apoptosis in heatstroke mice. Cell Mol Biol (Noisy-le-grand) 64: 36-42, 2018.

20. Geng Y, Ma Q, Liu YN, Peng N, Yuan FF, Li XG, Li M, Wu YS, $\mathrm{Li} \mathrm{BL}$, Song WB, et al: Heatstroke induces liver injury via IL-1 $\beta$ and HMGB1-induced pyroptosis. J Hepatol 63: 622-633, 2015.

21. Ji J, Hong X, Su L and Liu Z: Proteomic identification of hippocalcin and its protective role in heatstroke-induced hypothalamic injury in mice. J Cell Physiol 234: 3775-3789, 2019.

22. Hsu YL, Yu HS, Lin HC, Wu KY, Yang RC and Kuo PL: Heat shock induces apoptosis through reactive oxygen species involving mitochondrial and death receptor pathways in corneal cells. Exp Eye Res 93: 405-412, 2011.

23. Milleron RS and Bratton SB: Heat shock induces apoptosis independently of any known initiator caspase-activating complex J Biol Chem 281: 16991-17000, 2006.

24. North S and Hainaut P: P53 and cell cycle control: A finger in every pie. Pathol Biol (Paris) 48: 255-270, 2000

25. Ye F, Deng PY, Li D, Luo D, Li NS, Deng S, Deng HW and $\mathrm{Li}$ YJ: Involvement of endothelial cell-derived CGRP in heat stress-induced protection of endothelial function. Vasc Pharmacol 46: 238-246, 2007.

26. Kerr JF, Wyllie AH and Currie AR: Apoptosis: A basic biological phenomenon with wide-ranging implications in tissue kinetics. Br J Cancer 26: 239-257, 1972.
27. Jeong SY, Seol DW: The role of mitochondria in apoptosis. BMB Rep 41: 11-22, 2008.

28. Santra S, Kaittanis C and Perez JM: Cytochrome C encapsulating theranostic nanoparticles: A novel bifunctional system for targeted delivery of therapeutic membrane-impermeable proteins to tumors and imaging of cancer therapy. Mol Pharm 7: $1209-1222,2010$

29. Yamada $\mathrm{Y}$ and Harashima H: Mitochondrial drug delivery systems for macromolecule and their therapeutic application to mitochondrial diseases. Adv Drug Deliv Rev 60: 1439-1462, 2008.

30. Wang Y, Xia C, Lun Z, Lv Y, Chen W and Li T: Crosstalk between p38 MAPK and caspase-9 regulates mitochondria-mediated apoptosis induced by tetra- $\alpha$-(4-carboxyphenoxy) phthalocyanine zinc photodynamic therapy in LoVo cells. Oncol Rep 39 61-70, 2018.

31. Chen YJ, Liu WH, Kao PH, Wang JJ and Chang LS: Involvement of p38 MAPK- and JNK-modulated expression of Bcl-2 and Bax in Naja nigricollis CMS-9-induced apoptosis of human leukemia K562 cells. Toxicon 55: 1306-1316, 2010.

32. Deng YT, Huang HC and Lin JK: Rotenone induces apoptosis in MCF07 human breast cancer cell-mediated ROS through JNK and p38 signaling. Mol Carcinog 49: 141-151, 2010.

33. Kang YH and Lee SJ: The role of p38 MAPK and JNK in Arsenic trioxide-induced mitochondrial cell death in human cervical cancer cells. J Cell Physiol 217: 23-33, 2008.

34. Su JC, Lin KL, Chien CM, Lu CM, Chen YL, Chang LS and Lin SR: Novel indoloquinoline derivative, IQDMA, induces $\mathrm{G}(2) / \mathrm{M}$ phase arrest and apoptosis in A549 cells through JNK/p38 MAPK signaling activation. Life Sci 85: 505-516, 2009.

(i) $\odot$ This work is licensed under a Creative Commons Attribution-NonCommercial-NoDerivatives 4.0 International (CC BY-NC-ND 4.0) License. 